\title{
HETEROGENEIDADE E DINÂMICAS DAS FONTES DE OCUPAÇÃO E RENDA DAS FAMÍLIAS RURAIS NOS ESTADOS DO NORDESTE BRASILEIRO
}

\author{
HETEROGENEITY AND DYNAMICS OF THE SOURCES OF OCCUPATION \\ AND INCOME OF RURAL FAMILIES IN THE STATES OF BRAZILIAN \\ NORTHEAST
}

\section{HETEROGENEIDAD Y DINÁMICA DE LAS FUENTES DE OCUPACIÓN E INGRESOS DE LAS FAMILIAS RURALES EN LOS ESTADOS DEL NORESTE BRASILEÑO}

\author{
Joacir Rufino de Aquino ${ }^{1}$ \\ https://orcid.org/0000-0003-0772-7141 \\ Carlos Alves do Nascimento ${ }^{2}$ \\ https://orcid.org/0000-0003-3495-8076
}

Submissão: 26/04/2020 / Aceito: 17/05/2020

\begin{abstract}
RESUMO
O objetivo deste artigo é analisar a heterogeneidade e as dinâmicas das fontes de ocupação e renda das famílias rurais dos estados nordestinos durante a Grande Seca que atingiu a região (2012-2015). Para tanto, utilizou-se como referência informações obtidas a partir do processamento dos microdados da Pesquisa Nacional por Amostra de Domicílios (PNAD), do IBGE. Em linhas gerais, os dados analisados mostram que, apesar dos impactos da estiagem prolongada, as áreas rurais dos estados do Nordeste não apresentaram decrescimento populacional como em épocas passadas. Esse resultado é explicado, em parte, pela diversificação das fontes de ocupação e renda das famílias rurais, que estão deixando de depender exclusivamente da agropecuária para sobreviver e se tornando cada vez mais pluriativas, não agrícolas ou, simplesmente, convertendo o campo em local de moradia. Tal processo, que apresenta dinâmicas diferenciadas entre os estados da região, tem sido facilitado no período recente pela maior sinergia entre as áreas rurais e urbanas proporcionada pela democratização dos meios de transporte e de comunicação, bem como pela rede de proteção social criada pelas políticas públicas de transferência de renda e de desenvolvimento rural.
\end{abstract}

Palavras-chave: Atividades não agrícolas; Famílias Rurais; Pluriatividade; Seca; Nordeste.

\begin{abstract}
The objective of this article is to analyze the heterogeneity and dynamics of the sources of occupation and income of rural families in the northeastern states during the Great Drought that hit the region (2012-2015). For this, information obtained from the processing of microdata from the National Household Sample Survey (PNAD), from IBGE, was used as a reference. In general, the data analyzed show that, despite the impacts of the prolonged drought, the rural areas of the Northeastern states did not show population decrease as in past times. This result is explained, in part, by the diversification of the sources of occupation and income of rural families, who are no longer relying exclusively on agriculture to survive and becoming increasingly pluriactive, non-agricultural or, simply, converting the countryside into a place of housing. This process, which presents different dynamics between the states of the region, has been facilitated in the recent period by the greater synergy between rural and urban areas provided by the democratization of means of transport and
\end{abstract}

\footnotetext{
${ }^{1}$ Mestre em Economia Rural e Regional pela Universidade Federal de Campina Grande (2003). Atualmente é Professor Adjunto IV na Universidade do Estado do Rio Grande do Norte (Campus de Assú).

${ }^{2}$ Doutorado em Economia Aplicada pela Universidade Estadual de Campinas (2005). Atualmente é Professor do Programa de Pós-Graduação do Instituto de Economia da Universidade Federal de Uberlândia.
} 
communication, as well as by the social protection network created by public policies of income transfer and rural development.

Keywords: Non-agricultural activities; Rural Families; Pluriactivity; Drought; Northeast.

\section{RESUMEN}

El objetivo de este artículo es analizar la heterogeneidad y la dinámica de las fuentes de ocupación e ingresos de las familias rurales en los estados del noreste durante la Gran Sequía que azotó la región (2012-2015). Para ello, se utilizó como referencia la información obtenida del procesamiento de microdatos de la Encuesta Nacional de Muestra de Hogares (PNAD), del IBGE. En general, los datos analizados muestran que, a pesar de los impactos de la sequía prolongada, las áreas rurales de los estados del noreste no mostraron una disminución de la población como en el pasado. Este resultado se explica, en parte, por la diversificación de las fuentes de ocupación e ingresos de las familias rurales, que ya no dependen exclusivamente de la agricultura para sobrevivir y se vuelven cada vez más pluriactivas, no agrícolas o, simplemente, convierten el campo en un lugar de vivienda. Este proceso, que presenta diferentes dinámicas entre los estados de la región, se ha visto facilitado en el período reciente por la mayor sinergia entre las zonas rurales y urbanas que proporciona la democratización de los medios de transporte y comunicación, así como por la red de protección social creada por las políticas públicas de transferencia de ingresos y desarrollo rural.

Palabras-chave: Actividades no agrícolas; Familias rurales; Pluriactividad; Sequía; Noreste

\section{INTRODUÇÃO}

As secas sempre trouxeram à tona as fragilidades e contradições do modelo agrícola nordestino. Na literatura, basta lembrar as histórias contadas em livros clássicos, como $\mathrm{O}$ Quinze (QUEIROZ, 2012), Vidas Secas (RAMOS, 2008) e Morte e Vida Severina (MELO NETO, 2007). Todas essas obras, de significativa expressão nacional, retratam a calamidade social em períodos de seca, com levas de pessoas flageladas migrando do campo e outras tendo suas vidas ceifadas pela falta de água e pela fome.

No final dos anos 1950, no conhecido documento do Grupo de Trabalho para o Desenvolvimento do Nordeste (GTDN), Celso Furtado mostrou que o problema maior da seca no Nordeste era decorrente da brutal desigualdade na distribuição da posse da terra vigente na região e da dependência das famílias rurais das atividades agropecuárias de sequeiro para garantir seu alimento e uma reduzida renda monetária (FURTADO, 1998; GTDN, 1967).

Transcorridos mais de 60 anos do diagnóstico do GTDN, que serviu de base para criação da SUDENE em 1959, percebe-se que o Nordeste vivenciou transformações marcantes em sua estrutura produtiva. A agropecuária reduziu sua importância em todos os estados, enquanto a indústria e, especialmente, os setores de comércio e serviços localizados nas áreas urbanas passaram a representar as maiores parcelas do PIB regional. 
E as secas, deixaram de acontecer? Não, elas continuam sendo "repetitivas, previsíveis e inevitáveis" (MAIA GOMES, 2001), mas parece que seus efeitos catastróficos sobre a população têm sido amortecidos.

De fato, no período de 2012 a 2017, a região registrou umas das mais severas estiagens dos últimos 100 anos e ela revelou mudanças importantes em relação aos episódios retratados nas obras literárias citadas anteriormente. É bem verdade que a Grande Seca, como será designada doravante, provocou impactos negativos graves nas atividades agropecuárias, nas economias locais e no meio ambiente. Mesmo assim, não foram registrados casos de migração, saques nos comércios ou mortes de gente faminta como ocorria até o começo da última década do século XX (IBGE, 2018; MARTINS; MAGALHÃES, 2015; NYS; ENGLE; MAGALHÃES, 2016).

Para alguns especialistas em economia regional, a Grande Seca "sem migração" é fruto das melhorias na infraestrutura hídrica (açudes, barragens, cisternas etc.) e da expansão da rede de proteção social criada pelas políticas públicas federais a partir dos anos 2000, que, em conjunto com as ações governamentais de apoio a economia em geral, aumentou a resiliência dos habitantes do campo e das cidades nordestinas (ARAÚJO, 2014; CARVALHO, 2014; MAIA GOMES, 2014).

Outros trabalhos recentes mostram que além desses aspectos, do ponto de vista dos dados agregados para a região, a explicação está atrelada às múltiplas alternativas que as famílias rurais têm encontrado para sobreviver, diversificando suas estratégias de reprodução social através da pluriatividade, de ocupações não agrícolas e de rendas não advindas da produção (AQUINO; NASCIMENTO, 2020). No entanto, como lembram Araújo (2000, 2013) e Gomes da Silva e Veras (2001), a região Nordeste apresenta uma marcante diferenciação interna. Desse modo, é preciso avançar na discussão para além da média regional, desagregando os indicadores ao nível estadual, sob pena de se comprometer o entendimento da diversidade das situações locais.

Assim sendo, visando contribuir com os debates sobre a temática, o objetivo do presente artigo é analisar a heterogeneidade e as dinâmicas das fontes de ocupação e renda das famílias rurais nos nove estados nordestinos durante parte da estiagem prolongada que atingiu a região (2012-2015). Para tanto, utilizou-se como referência informações obtidas a partir do processamento dos microdados da Pesquisa Nacional por Amostra de Domicílios (PNAD), do IBGE. 
A estrutura do texto foi dividida em mais três seções, além desta breve Introdução. Na segunda seção, discute-se de forma sucinta os procedimentos metodológicos adotados no processamento dos dados secundários que serão utilizados no estudo. A terceira seção apresenta e discute os resultados obtidos por meio da análise dos dados estatísticos tabulados. A última seção, por sua vez, faz uma breve síntese dos achados da pesquisa e sinaliza alguns insights para novas investigações na área.

\section{METODOLOGIA}

As informações que serão analisadas neste estudo foram organizadas objetivando identificar os tipos e subtipos de famílias residentes nas áreas rurais das nove Unidades Federativas (UFs) que constituem a região Nordeste a partir da ocupação de seus membros. As famílias foram classificadas, em primeiro lugar, segundo a posição na ocupação: empregadora, conta-própria, trabalhadores empregados e não ocupados, na semana de referência da Pesquisa Nacional por Amostra de Domicílios (PNAD), do IBGE. ${ }^{3}$

A metodologia de construção dos tipos de famílias deixa de fora da contagem os pensionistas, os empregados domésticos e os parentes dos empregados domésticos. Com isso, a unidade de análise adotada são as "famílias extensas", que compreendem, além da família nuclear, os parentes desta que vivem no mesmo domicílio (não obstante formem outro casal) e os agregados. ${ }^{4}$

As famílias extensas foram divididas segundo o ramo de atividades em que seus membros estavam envolvidos (agropecuárias, não agropecuárias e pluriativas), conforme os critérios descritos a seguir:

i) as famílias agropecuárias são aquelas em que pelo menos um de seus membros estava envolvido em atividades agropecuárias - e nenhum outro ocupado em atividades não agropecuárias - como sua ocupação principal na semana de

\footnotetext{
${ }^{3}$ A base de dados utilizada para a construção da tipologia aqui adotada é oriunda dos microdados das PNADs devidamente atualizados conforme os novos critérios de "reponderação" adotados pelo IBGE/SIDRA (2015). As informações dessa pesquisa amostral são obtidas pelos técnicos do IBGE tomando como período de referência para a coleta das informações a última semana do mês de setembro de cada ano civil.

${ }^{4}$ Os critérios de agrupamento empregados seguem a metodologia utilizada originalmente nos trabalhos do Projeto RURBANO, coordenado pelo professor José Graziano da Silva da UNICAMP (Cf. GRAZIANO DA SILVA, 1999). Os fundamentos teóricos dessa escolha metodológica, por seu turno, encontram-se sistematizados em Mattei (2007).
} 
referência da pesquisa (normalmente a última ou a penúltima semana de setembro de cada ano);

ii) foram consideradas famílias não agropecuárias, em contraste com os critérios anteriores, as que pelo menos um de seus membros exercia atividades não agropecuárias - e nenhum outro estava ocupado em atividades agropecuárias; e

iii) classificou-se como famílias pluriativas aquelas em que pelo menos um de seus membros exercia alguma atividade agropecuária e pelo menos um outro exercia uma atividade não agropecuária; e

iv) no caso de nenhum dos membros da família ter-se declarado ocupado, na semana de referência da PNAD, a família foi considerada como família de não ocupados.

Cabe destacar que, nas informações relativas às pessoas (com 10 anos ou mais de idade) residentes nas áreas rurais nordestinas, à semelhança dos tipos de famílias, também haverá a divisão entre os ocupados segundo o ramo de atividade (população economicamente ativa - PEA rural, agropecuária e não agropecuária) e a população de não ocupados - formada pela PEA rural não ocupada (desempregados) e os inativos (com e sem aposentadoria/ pensão).

O período de análise será de 2011 a 2015, ou seja, com abrangência menor do que o tempo de duração da Grande Seca (2012-2017). O ano de 2011, considerado de inverno normal no Nordeste, foi incluído no estudo para fins de comparação com os anos secos a partir de 2012. Já o limite da série trabalhada restringe-se a 2015, unicamente por conta da indisponibilidade de estatísticas mais atualizadas depois dessa data no banco de microdados da PNAD/IBGE.

Em termos operacionais, registre-se que o procedimento empregado para verificar a ocorrência ou não de tendência estatística na evolução do número de famílias e de pessoas nas tabelas que serão apresentadas ao longo do texto é baseado em um modelo de regressão $\log$-linear contra o tempo ${ }^{5}$. O referido modelo, de acordo com a especificação proposta por Neder (2000), pode ser assim apresentado:

\footnotetext{
${ }^{5}$ O modelo de regressão log-linear, de acordo com Neder (2000), é utilizado para a determinação de taxas de crescimento ao longo do tempo e não apenas entre dois pontos extremos de uma série temporal, de modo que se evitam possíveis efeitos devidos a valores atípicos nas extremidades de uma série de tempo, que podem levar à subestimação ou superestimação da taxa de crescimento. Para obter maiores detalhes sobre a referida técnica estatística, com exemplos empíricos, ver: Gujarati (2006) e Hoffmann (2000).
} 
$\mathrm{Y}_{\mathrm{t}}=\varphi \mathrm{e}^{\theta \mathrm{t}}$

Onde:

$\mathrm{Y}_{\mathrm{t}}=\mathrm{o}$ valor da população para $\mathrm{o}$ ano $\mathrm{t}$

$\varphi=$ um parâmetro a ser estimado da regressão

$\theta=$ um parâmetro a ser estimado da regressão

$\mathrm{e}=\mathrm{a}$ base dos logaritmos naturais

$\mathrm{t}=\mathrm{o}$ tempo (ano da PNAD; $\mathrm{t}=2011,2012, \ldots, 2015)$

Uma vez que o modelo estatístico em foco é não-linear, para transformá-lo em um modelo de regressão linear, aplica-se o logaritmo natural nos dois lados da expressão, obtendo-se a seguinte fórmula:

$\ln Y_{t}=\ln \varphi+\theta t \quad$ ou $\quad Y^{\prime}{ }_{t}=\varphi^{\prime}+\theta t$

Em que:

$\ln \mathrm{Y}_{\mathrm{t}}=\mathrm{Y}^{\prime}{ }_{\mathrm{t}}$

$\ln \varphi=\varphi^{\prime}$

$\theta=$ estimativa do coeficiente de regressão

Como resultado do método delineado, a taxa de crescimento média ao ano estimada para o período em análise, ou seja, não apenas em um ponto do tempo, foi igual a:

$$
\left[\left(\mathrm{e}^{\theta}-1\right) \times 100\right]
$$

Por fim, utilizou-se o teste t para averiguar a significância do parâmetro $\theta$, pois pode ocorrer que o teste t para a hipótese de que $\theta=0$ não seja significativo, implicando, nesse caso $(\theta=0$ ou e $\theta=1)$, ao nível de significância adotado, que os dados não permitem rejeitar a hipótese de que Yt não tem tendência de crescimento ou decrescimento ao longo da série de tempo observada. Ocorrendo $\theta>0$ ou $\theta<0$, pode-se falar, então, em tendência de crescimento ou decrescimento ao longo da série de tempo observada (HOFFMANN, 2000).

A partir desses procedimentos metodológicos, apresentados brevemente aqui, é que foi elaborada a base de dados que balizará a investigação proposta. Tais dados permitem observar o perfil das formas de ocupação e renda das famílias rurais das UFs nordestinas e verificar as tendências e as características ocupacionais da população residente neste espaço geográfico particular. É o que se procurará mostrar na próxima seção. 


\section{RESULTADOS E DISCUSSÃO}

No limiar do século XXI, uma parcela expressiva das famílias do Nordeste rural ainda vive em grande dificuldade socioeconômica comparativamente as demais regiões do país. Os índices de pobreza também continuam altos, apesar da queda recente (BURITI; BARBOSA, 2018; MELLO, 2018; SOARES, 2016). Contudo, o campo nordestino não apresenta sinais de esvaziamento acelerado e, inclusive, tem registrado taxas de crescimento demográfico positivas. Esse processo é fruto de mudanças notáveis na estrutura ocupacional da população em relação ao passado, apresentando dinâmicas diferenciadas entre os estados da região.

Para ilustrar o argumento acima, a Tabela 1 mostra a distribuição e evolução dos contingentes totais de famílias rurais nordestinas (Nordeste e suas Unidades da Federação UFs), entre 2011 e 2015. Uma primeira observação importante é a de que, no período dentro do qual as famílias rurais residentes nas UFs nordestinas estão sendo analisadas, correspondente à última grave seca que se abateu sobre a região, nenhuma UF nordestina registrou redução do número de famílias rurais totais. Pelo contrário, todas elas, sem exceção, experimentaram crescimento significativo (sentido estatístico) ${ }^{6}$ no total de famílias.

Os estados que se destacaram com o crescimento do conjunto das suas respectivas famílias rurais, à taxas médias anuais acima da média Nordestina (3,2\%a.a.), foram os do Ceará (4,1\%a.a.), Rio Grande do Norte (4,5\%a.a.), Pernambuco (4,5\%a.a.) e Sergipe (4,4\%a.a.). O crescimento dos contingentes familiares rurais dos demais estados nordestinos ficou abaixo da média da região, mas praticamente todos com taxas positivas expressivas e significativas.

\footnotetext{
${ }^{6} \mathrm{O}$ qualificativo significativo concerne ao teste estatístico feito para verificar se a evolução dos dados (número de famílias) apresenta alguma tendência, de crescimento ou de redução. Dessa forma, sempre que for usado doravante esse qualificativo (representado pelos asteriscos que acompanham algumas taxas nas tabelas) a intenção será a de mostrar que os dados apresentam uma certa tendência estatística, de modo que nos casos em que as taxas de crescimento das tabelas não estiverem acompanhadas de asteriscos significará que as mesmas não são estatisticamente significativas e, portanto, não representam nenhuma tendência - o que poderá ser traduzido, por conseguinte, como uma estabilidade na evolução dos dados.
} 
Tabela 1 - Evolução (e taxas médias de crescimento) dos contingentes totais de famílias extensas residentes rurais: UFs e Nordeste, 2011 a 2015 ( $\mathrm{N}^{\circ}$ Famílias x 1.000)

\begin{tabular}{|c|c|c|c|c|c|c|c|}
\hline UFs Nordestinas & 2011 & 2012 & 2013 & 2014 & 2015 & \multicolumn{2}{|c|}{$\begin{array}{c}\Delta \% \text { a.a. } \\
2011 / 2015^{a}\end{array}$} \\
\hline Maranhão & 682 & 728 & 745 & 740 & 774 & 2,7 & 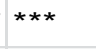 \\
\hline Piauí & 293 & 300 & 297 & 309 & 311 & 1,5 & 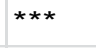 \\
\hline Ceará & 659 & 653 & 694 & 714 & 771 & 4,1 & $* \star \star$ \\
\hline Rio Gde Norte & 201 & 197 & 209 & 242 & 227 & 4,5 & $\star \star$ \\
\hline Paraíba & 208 & 222 & 234 & 222 & 242 & 3,1 & ** \\
\hline Pernambuco & 452 & 491 & 500 & 520 & 547 & 4,5 & $* \star *$ \\
\hline Alagoas & 240 & 254 & 266 & 258 & 260 & 1,8 & * \\
\hline Sergipe & 169 & 171 & 187 & 193 & 198 & 4,4 & $* \star *$ \\
\hline Bahia & 1.108 & 1.165 & 1.176 & 1.187 & 1.252 & 2,7 & $\star \star \star *$ \\
\hline Nordeste & 4.012 & 4.181 & 4.308 & 4.384 & 4.581 & 3,2 & $* \star *$ \\
\hline
\end{tabular}

Fonte: Microdados das PNADs/IBGE. Elaboração dos autores.

Nota: O (a) representa a estimativa do coeficiente de uma regressão log-linear contra o tempo. Neste caso, o teste $\mathrm{t}$ indica a existência ou não de uma tendência nos dados. Já os asteriscos (***, **, *) representam os níveis de significância de 5\% (***), 10\% (**) e 20\% (*).

Os dados informados na Tabela 2 objetivam destacar a nítida heterogeneidade social das famílias rurais em todos os estados pesquisados, do ponto de vista dos ramos de atividade nos quais as famílias estão ocupadas e a partir dos quais as mesmas são classificadas como agropecuárias, pluriativas e não agropecuárias, assim como também estão sendo consideradas as famílias de não ocupados.

Pode-se observar que, em 2015, das nove UFs nordestinas, em seis delas praticamente $60,0 \%$ ou mais das suas famílias rurais não eram exclusivamente famílias agropecuárias - as três exceções eram Maranhão, Piauí e Bahia, que lograram elevar a média regional, nessa questão, para um pouco acima dos 40,0\%. O estado do Rio Grande do Norte era o que proporcionalmente menos abrigava as famílias exclusivamente agropecuárias $(32,7 \%)$, revelando o aprofundamento de um processo diferenciado de “êxodo agrícola e não rural” já identificado em outros estudos (AQUINO; NASCIMENTO, 2015; GOMES DA SILVA, 2000).

Além dessas observações, compete destacar que em quatro UFs (CE, RN, PE e AL) mais de 40,0\% das famílias rurais não têm nenhum vínculo direto com as atividades agropecuárias, ou seja, quase a metade das famílias rurais são não agropecuárias ou não ocupadas. Com a única exceção do Piauí (27,4\%), todas as demais UFs abrigavam em seus respectivos rurais praticamente mais de um terço do conjunto desses dois grupos familiares, em 2015. 
Tabela 2 - Participação relativa (\%) de cada um dos tipos de famílias (segundo o ramo de atividade) na totalidade das famílias rurais: UFs e Nordeste, 2015

\begin{tabular}{|c|c|c|c|c|}
\hline \multirow[b]{2}{*}{ UFs Nordestinas } & \multicolumn{4}{|c|}{ TIPOS DE FAMÍLIAS } \\
\hline & Agropecuárias & Pluriativas & $\begin{array}{c}\text { Não } \\
\text { Agropecuárias }\end{array}$ & Não Ocupadas \\
\hline Maranhão & 49,2 & 15,9 & 23,1 & 11,7 \\
\hline Ceará & 39,0 & 14,1 & 22,3 & 24,6 \\
\hline Rio Gde Norte & 32,7 & 15,6 & 34,3 & 17,4 \\
\hline Alagoas & 37,0 & 8,7 & 21,8 & 32,4 \\
\hline Sergipe & 40,3 & 22,1 & 25,0 & 12,6 \\
\hline Bahia & 48,8 & 15,5 & 17,0 & 18,7 \\
\hline Nordeste & 43,7 & 16,3 & 21,8 & 18,2 \\
\hline
\end{tabular}

Fonte: Microdados das PNADs/IBGE. Elaboração dos autores.

Note-se que as possíveis explicações para o crescimento generalizado dos contingentes familiares rurais nordestinos totais variam de uma UF para outra. A heterogeneidade dos casos pode ser captada na Tabela 3, a seguir, ao se observar as trajetórias das famílias classificadas pelos ramos de atividade em que se ocupam.

Nesse sentido, uma primeira observação importante é a de que o único caso de homogeneidade entre as UFs é o de que, generalizadamente, em todas elas (e no Nordeste como um todo), as famílias não agropecuárias residentes rurais exibiram trajetória de crescimento significativo (sentido estatístico), contribuindo inegavelmente para aquele desempenho positivo dos totais familiares rurais registrado na Tabela 1. As famílias pluriativas, por sua vez, contribuíram (com taxas estatisticamente significativas) para o crescimento dos totais familiares rurais apenas nos estados do RN, PB, PE e SE. Já as famílias de não ocupadas somente deram essa contribuição nos estados do CE, AL e BA.

Tabela 3 - Taxas médias de crescimento (\%a.a.) dos diferentes tipos de famílias extensas residentes rurais: UFs e Nordeste, 2011 a 2015

\begin{tabular}{|c|c|c|c|c|c|c|c|c|}
\hline \multirow{3}{*}{$\begin{array}{l}\text { UFs Nordestinas } \\
\text { Maranhão }\end{array}$} & \multicolumn{8}{|c|}{ TIPOS DE FAMÍLIAS } \\
\hline & \multicolumn{2}{|c|}{ Agropecuárias } & \multicolumn{2}{|c|}{ Pluriativas } & \multicolumn{2}{|c|}{$\begin{array}{c}\text { Não } \\
\text { Agropecuárias }\end{array}$} & \multicolumn{2}{|c|}{ Não Ocupadas } \\
\hline & 2,2 & ** & 2,5 & & 3,7 & *** & 3,4 & \\
\hline Ceará & $-2,9$ & & $-2,1$ & & 14,8 & $\star * \star$ & 19,9 & ** \\
\hline Rio Gde Norte & $-2,2$ & & 6,1 & * & 10,6 & $\star \star$ & 7,4 & \\
\hline Alagoas & $-6,3$ & $\star \star \star *$ & $-11,0$ & ** & 11,4 & * & 19,8 & $\star \star \star *$ \\
\hline Sergipe & 1,5 & ** & 12,4 & $\star * *$ & 3,3 & * & 4,5 & \\
\hline Bahia & 0,1 & & 0,8 & & 7,1 & $* * *$ & 8,9 & $\star * *$ \\
\hline Nordeste & $-0,5$ & & 1,9 & * & 8,2 & $* * *$ & 10,3 & ** \\
\hline
\end{tabular}

Fonte: Microdados das PNADs/IBGE. Elaboração dos autores.

Nota: O (a) representa a estimativa do coeficiente de uma regressão log-linear contra o tempo. Neste caso, o teste $\mathrm{t}$ indica a existência ou não de uma tendência nos dados. Já os asteriscos (***, **, *) representam os níveis de significância de $5 \%(* * *), 10 \%(* *)$ e $20 \%(*)$. 
Outro aspecto importante da heterogeneidade é o de que os dois únicos estados em que as famílias agropecuárias também contribuíram para o crescimento dos totais familiares rurais, foram Maranhão e Sergipe. Na maioria dos demais estados, esse grupo familiar, na verdade, contrariamente aos outros três grupos familiares, os dados da Tabela 3 sugerem que contribuiu para conter, com suas taxas negativas - embora apenas uma, em Alagoas, tenha sido significativa (sentido estatístico) -, o crescimento dos totais familiares rurais nordestinos.

Analisando a mesma questão pelo ângulo não mais das famílias, mas dos totais de pessoas com 10 anos ou mais de idade residentes nas UFs nordestinas, a Tabela 4 revela de forma análoga às observações relativas aos contingentes totais de famílias (Tabela 1) - o aumento significativo (sentido estatístico) do total da população rural nordestina a uma taxa média anual de 1,8\%a.a., no período analisado. Em outras palavras, não obstante o grave período de crise hídrica ensejada pela Grande Seca, nenhuma UF nordestina registrou taxa negativa de crescimento de suas populações rurais - cinco delas exibiram taxas positivas significativas (sentido estatístico), sendo que quatro entre as mesmas (MA, RN, PE, SE) experimentaram taxa de crescimento acima da média da região.

Tabela 4 - Evolução (e taxas de crescimento) dos contingentes totais de pessoas residentes rurais: UFs e

\begin{tabular}{|c|c|c|c|c|c|c|c|}
\hline \multirow{2}{*}{$\begin{array}{l}\text { UFs Nordestinas } \\
\text { Maranhão }\end{array}$} & \multirow{2}{*}{$\frac{2011}{2.082}$} & \multirow{2}{*}{$\frac{2012}{2.146}$} & \multirow{2}{*}{$\begin{aligned} 2013 \\
2.246\end{aligned}$} & \multirow{2}{*}{$\begin{array}{r}2014 \\
2.239\end{array}$} & \multirow{2}{*}{$\frac{2015}{2.268}$} & \multicolumn{2}{|c|}{$\begin{array}{c}\Delta \% \text { a.a. } \\
2011 / 2015^{a}\end{array}$} \\
\hline & & & & & & 2,2 & $\star \star \star *$ \\
\hline Ceará & 1.970 & 1.956 & 2.004 & 1.973 & 2.078 & 1,2 & * \\
\hline Rio Gde Norte & 598 & 577 & 611 & 681 & 663 & 3,8 & $\star \star$ \\
\hline Alagoas & 748 & 767 & 769 & 788 & 744 & 0,2 & \\
\hline Sergipe & 465 & 481 & 493 & 527 & 547 & 4,3 & $* * *$ \\
\hline Bahia & 3.227 & 3.264 & 3.291 & 3.194 & 3.364 & 0,6 & \\
\hline Nordeste & 11.870 & 12.123 & 12.349 & 12.367 & 12.855 & 1,8 & $\star \star \star *$ \\
\hline
\end{tabular}

Fonte: Microdados das PNADs/IBGE. Elaboração dos autores.

Nota: O (a) representa a estimativa do coeficiente de uma regressão log-linear contra o tempo. Neste caso, o teste $\mathrm{t}$ indica a existência ou não de uma tendência nos dados. Já os asteriscos $(* * *, * *, *)$ representam os níveis de significância de 5\% (***), 10\% (**) e 20\% (*).

Quanto à distribuição da população rural nordestina pela ótica dos ramos de atividades nos quais se encontra ocupada, assim como também pelo prisma da não ocupação, a Tabela 5 - comparativamente ao que foi destacado em relação às famílias (Tabela 1) - é mais enfática em desnudar o pequeno peso relativo do vínculo direto da população rural às atividades agropecuárias. Praticamente todas as UFs nordestinas - única exceção o Piauí - tinham menos de 40,0\% de suas populações rurais com algum vínculo 
produtivo nas atividades agropecuárias - sendo que três estados (CE, RN e AL) registraram menos de 30,0\%, em 2015.

Chama à atenção a forte presença da população inativa residente nas áreas rurais das UFs nordestinas. Em quase todas elas a população inativa representa mais de $40,0 \%$ da população rural - as únicas exceções são os estados do Piauí $(31,8 \%)$ e de Sergipe $(37,1 \%)$ -, sendo que no Ceará os inativos rurais ultrapassam 50,0\% enquanto em Alagoas superam os 60,0\%. Considerando também os percentuais da PEA rural não ocupada, essas informações expressam a importância das áreas rurais nordestinas como local essencialmente de moradia para expressivas parcelas da população. Adicionando a esses percentuais a participação também expressiva da PEA rural não agropecuária, todas essas informações podem estar revelando que as demandas (referentes à trabalho, moradia, educação, lazer, infraestrutura social, etc.) da população rural - em todas as UFs nordestinas - são mais amplas do que as demandas exclusivamente ligadas às atividades agropecuárias.

Tabela 5 - Distribuição (\%) da população rural, com 10 anos ou mais de idade, ocupada (segundo o ramo de atividade) e não ocupada: UFs e Nordeste, 2015

\begin{tabular}{|c|c|c|c|c|c|}
\hline \multirow[b]{2}{*}{ UFs Nordestinas } & \multicolumn{3}{|c|}{ PEA rural } & \multicolumn{2}{|c|}{ INATIVOS } \\
\hline & Agropecuária & $\begin{array}{c}\text { Não } \\
\text { Agropecuária }\end{array}$ & Não Ocupada & $\begin{array}{c}\text { Com } \\
\text { Aposent./Pensão }\end{array}$ & $\begin{array}{c}\text { Sem } \\
\text { Aposent./Pensão }\end{array}$ \\
\hline Maranhão & 37,4 & 18,4 & 2,0 & 8,7 & 33,5 \\
\hline Piauí & 45,6 & 21,2 & 1,4 & 8,4 & 23,4 \\
\hline Ceará & 28,3 & 17,6 & 2,0 & 14,7 & 37,4 \\
\hline Rio Gde Norte & 24,2 & 23,3 & 4,3 & 11,5 & 36,7 \\
\hline Paraíba & 35,0 & 21,8 & 3,0 & 11,8 & 28,4 \\
\hline Pernambuco & 30,8 & 19,3 & 3,1 & 12,6 & 34,2 \\
\hline Alagoas & 21,6 & 12,6 & 4,0 & 15,2 & 46,7 \\
\hline Sergipe & 36,8 & 22,8 & 3,3 & 8,1 & 29,0 \\
\hline Bahia & 38,3 & 14,9 & 2,5 & 12,8 & 31,5 \\
\hline Nordeste & 34,2 & 17,9 & 2,6 & 11,9 & 33,4 \\
\hline
\end{tabular}

Fonte: Microdados das PNADs/IBGE. Elaboração dos autores.

Dando continuidade à linha de raciocínio apresentada, a Tabela 6 possibilita levantar algumas evidências que ajudam a explicar o crescimento positivo dos totais populacionais (pessoas com 10 anos ou mais de idade) residentes nas áreas rurais da UFs nordestinas no período estudado, de grave seca. A análise dessa tabela revela a heterogeneidade vigente entre os rurais das UFs nordestinas no que respeita à evolução de suas populações economicamente ativas (PEAs) ocupadas em atividades agropecuárias. Observe-se que, enquanto três UFs (RN, PE e SE) registraram taxas positivas de crescimento de suas respectivas PEAs rurais agropecuárias, todas as demais exibiram taxas negativas de crescimento de suas PEAs rurais agropecuárias. 
Por outro lado, diferentemente dessas últimas observações, nota-se em todas as UFs rurais nordestinas (Tabela 6) uma generalizada homogeneidade de comportamento nas trajetórias de suas PEAs rurais ocupadas em atividades não agropecuárias. A única exceção, nesse aspecto, foi Alagoas que também registrou taxa positiva de crescimento, porém, não significativa (sentido estatístico).

Essas observações sugerem, portanto, mais uma vez (análogo ao que já havíamos conjecturado para o caso das famílias), que o recurso às atividades não agropecuárias ajuda a explicar o crescimento das populações rurais totais das UFs nordestinas. Mesmo nos três casos em que se observam taxas positivas de crescimento das PEAs rurais agropecuárias (RN, PE e SE), conforme apontado anteriormente, as taxas de crescimento das PEAs rurais não agropecuárias foram respectivamente bem mais intensas nessas mesmas três UFs.

Vale observar, ainda, que a evolução dos contingentes de pessoas não ocupadas residentes nas áreas rurais das UFs nordestinas (Tabela 6) ajuda a explicar o crescimento dos números totais de residentes rurais (Tabela 4), uma vez que as taxas de crescimento das populações não ocupadas foram positivas em cada uma das UFs. Contudo, em apenas quatro UFs (MA, CE, RN e AL) essas taxas foram significativas (sentido estatístico), reiterando que o destaque de melhor desempenho explicativo para o crescimento dos números totais de residentes rurais permanece com as PEAs rurais não agropecuárias, conforme comentado no parágrafo anterior. 
Tabela 6 - Taxas médias de crescimento (\%a.a.) da população rural ocupada (segundo o ramo de atividade) e

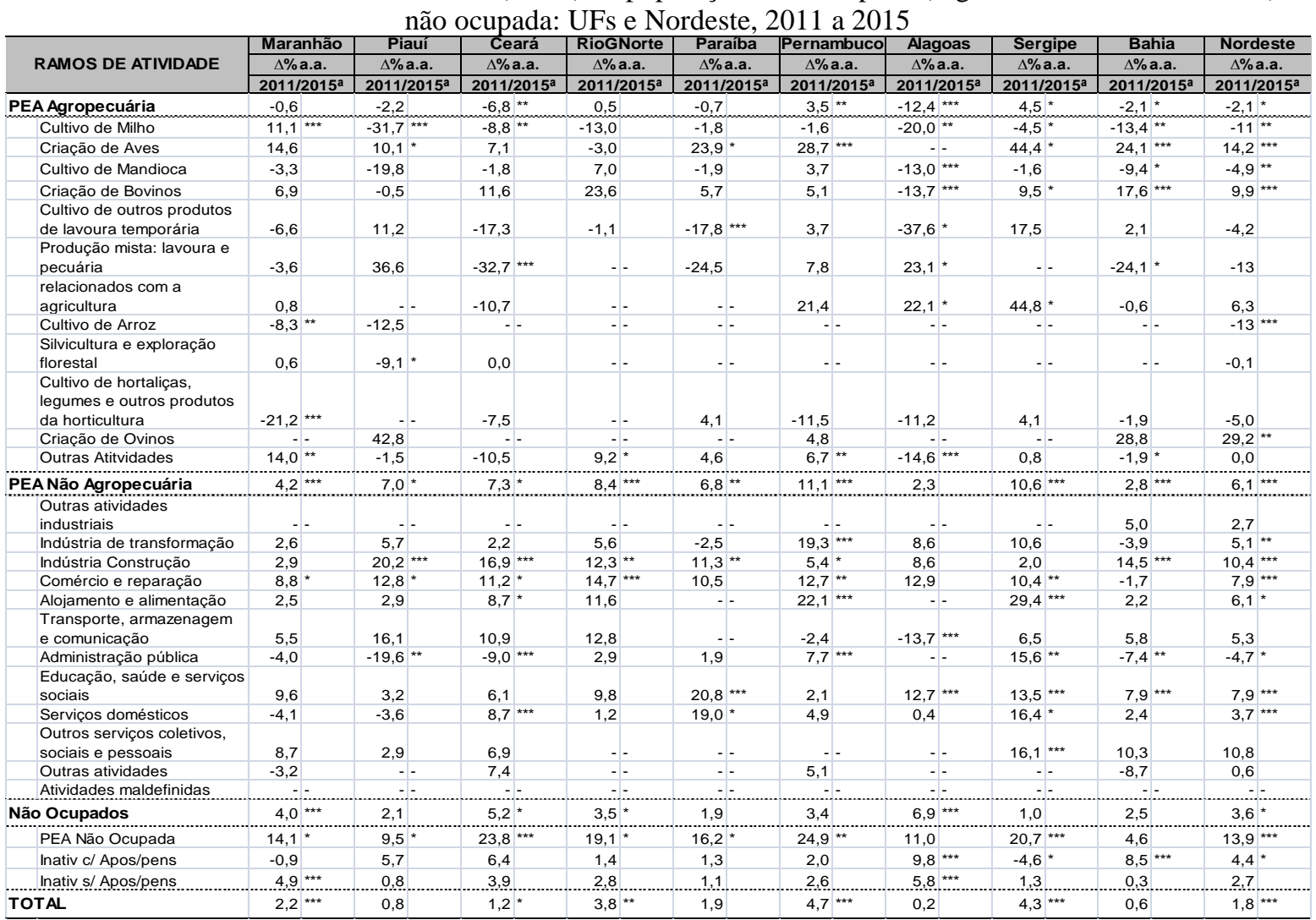

Fonte: Microdados das PNADs/IBGE. Elaboração dos autores.

Nota: Os dois traços (- -) significam os tipos de família com menos de 6 observações na amostra. Já (a) é estimativa do coeficiente de uma regressão log-linear contra o tempo. Neste caso, o teste t indica a existência ou não de uma tendência nos dados. Os asteriscos $(* * *, * *, *)$, por sua vez, representam os níveis de significância de $5 \%(* * *), 10 \%(* *)$ e $20 \%(*)$.

Com efeito, a Tabela 6 mostra também que, não obstante o registro de tendência de queda quase generalizada da PEA agropecuária - nas respectivas UFs - em lavouras tradicionais (especialmente milho e mandioca), verificou-se uma tendência quase generalizada de crescimento da ocupação principalmente nas atividades de criação de aves (maioria das UFs), seguida da criação de bovinos. Quanto à evolução da PEA rural não agropecuária, há uma diversidade maior de casos de atividades que experimentaram tendência de crescimento dessas ocupações comparativamente às atividades agropecuárias, reiterando as tendências anteriormente apontadas. $\mathrm{O}$ destaque principal, em virtude de uma maior generalidade de UFs, coube às ocupações em atividades na indústria da construção, comércio e reparação, alojamento e alimentação, educação, saúde e serviços sociais, e de certa forma também nos serviços domésticos remunerados.

No que tange ao crescimento generalizado de ocupações dos residentes rurais em atividades não agropecuárias, a base de dados utilizada - os microdados das PNADs/IBGE 
- não permite saber se tais ocupações são exercidas nas próprias áreas rurais ou em áreas urbanas. O que se pode constatar é que são residentes rurais ocupados em atividades não agropecuárias. Entretanto, a despeito de não se poder afirmar com certeza estatística qual o local (rural ou urbano) em que tais atividades são exercidas pelos residentes rurais, o que importa é compreender que uma parte dessas ocupações pode estar sendo realizada nas áreas rurais e outra parte sendo executada nas áreas urbanas.

A partir do exposto é possível supor a tendência crescente de um intenso movimento pendular semanal dos residentes rurais entre as duas áreas, rural e urbana, seja para exercer suas atividades de trabalho não agropecuárias (como é caso, por exemplo, dos trabalhadores que exercem atividades por conta própria ou como empregados) nas áreas urbanas, seja também apenas para realizar atividades escolares (caso dos jovens rurais) nessas mesmas áreas, mas, em ambas as situações, permanecendo com a residência no rural.

Tabela 7 - Distribuição e evolução do número de famílias rurais com e sem carro e/ou motocicleta: UFs e

\begin{tabular}{|c|c|c|c|c|c|c|c|c|c|c|c|c|c|c|c|c|c|c|c|c|c|c|c|}
\hline \multirow{2}{*}{$\begin{array}{l}\text { TIPO DE FAMILIA } \\
\text { Igropecuárias }\end{array}$} & \multicolumn{3}{|c|}{ Maranhão } & \multicolumn{3}{|c|}{ Piauí } & \multicolumn{2}{|c|}{ Ceará } & \multicolumn{2}{|c|}{ Rio G. do Norte } & \multicolumn{2}{|c|}{ Paraíba } & \multicolumn{2}{|c|}{ Pernambuco } & \multicolumn{2}{|c|}{ Alagoas } & \multicolumn{3}{|c|}{ Sergipe } & \multicolumn{2}{|r|}{ Bahia } & \multicolumn{2}{|c|}{ Nordeste } \\
\hline & \begin{tabular}{l|l|}
2015 & \\
100,0
\end{tabular} & & & 100,0 & & \multicolumn{2}{|c|}{2015 年 } & & $\frac{2015}{20}$ & & & & \begin{tabular}{l|l|}
2015 & \\
100,0
\end{tabular} & & 100,0 & & \multicolumn{2}{|c|}{\begin{tabular}{l|}
2015 \\
100,0
\end{tabular}} & & 100,0 & & 100,0 & \\
\hline Com Motocicleta & 43,8 & 10,8 & $8^{* * *}$ & 56,0 & 4,7 & & 45,0 & $4,7^{\star * *}$ & 50,5 & $13,1^{* * *}$ & 33,6 & $-1,6$ & 33,8 & $11,1^{* * *}$ & 32,0 & $7,2^{\text {** }}$ & & 3,6 & $7,5^{\text {*** }}$ & 32,0 & $11,6^{*}$ & 39,4 & $8,2^{* * *}$ \\
\hline Com Carro e Motocicleta & 2,2 & & -. & 7,1 & 9,8 & & 1,5 & 9,5 & 4,8 & & 7,9 & & 4,5 & 2,8 & 2,0 & 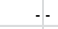 & & 4,1 & - & 5,6 & 16,2 * & 4,2 & $16,2^{\star \star *}$ \\
\hline Sem Carro e/ou Motocicleta & 50,7 & $-7,5$ & $5^{* * *}$ & 33,3 & $-7,2^{\star}$ & & 51,1 & $-3,5^{*}$ & 39,0 & $-11,2 * * *$ & 53,3 & $-1,6$ & 53,0 & $-4,6^{\text {t*x}}$ & 58,2 & $-4,1^{\text {* }}$ & & 7,2 & $-5,5^{\text {*** }}$ & 52,1 & $-6,6$ * & 49,9 & $-6,0^{* * *}$ \\
\hline Com Carro & 2,4 & & -. & 2,0 & & & 6,4 & .. & 10,0 & .. & 6,4 & .. & 13,9 & $5,4^{\star * *}$ & 16,7 & .. & & 9,2 & -. & 11,3 & 6,3 & 8,0 & 5,0 \\
\hline Com Motocicleta & 60,4 & 13,7 & $7^{* \star *}$ & 61,7 & 3,8 & & 49,6 & 3,1 & 38,0 & 2,2 & 57,7 & $7,6^{* * *}$ & 43,3 & 3,1 & 38,9 & 5,9 & & 11,2 & 1,8 & 43,7 & 8,2 & 48,9 & $5,8 * *$ \\
\hline Com Carro e Motocicleta & 7,9 & & $\cdots$ & 18,1 & 18,1 * & & 11,1 & $12,0^{*}$ & 12,0 & .. & 18,0 & .. & 14,2 & & 5,6 & .. & & 8,4 & -. & 12,5 & $12,0^{*}$ & 12,8 & $17,8^{\star * *}$ \\
\hline Sem Carro e/ou Motocicleta & 29,3 & $-14,7$ & $7^{\star \star \star *}$ & 18,1 & $-16,1$ * & & 32,9 & $-9,0^{*}$ & 40,0 & $-2,7$ & 17,9 & $-20,1^{* * *}$ & 28,5 & $-12,5^{* * *}$ & 38,9 & $-9,5^{\text {** }}$ & & 1,2 & $-7,6^{*}$ & 32,6 & $-10,6$ * & 30,3 & $-10,7^{\star * *}$ \\
\hline & & & & & & & 000 & & & & & & & & 1000 & & & & & 100,0 & & & \\
\hline Com Carro & $\begin{array}{r}100,0 \\
15,1\end{array}$ & 10,3 & $3^{\text {** }}$ & $\begin{array}{r}100,0 \\
7,8\end{array}$ & $-5,9$ & & 10,3 & $-2,8$ & $\begin{array}{r}100,0 \\
20,9\end{array}$ & $12,6^{*}$ & $\begin{array}{r}100,0 \\
9,9\end{array}$ & -. & $\begin{array}{r}100,0 \\
12,1\end{array}$ & $-8,2^{*}$ & $\begin{array}{r}100,0 \\
17,8\end{array}$ & $-0,3$ & & 3,3 & 4,1 & 19,6 & $8,2 *$ & $\begin{array}{r}14,6 \\
14,6\end{array}$ & 3,1 \\
\hline Não O & 100,0 & & & 100,0 & & & 00,0 & & 100,0 & & 100,0 & & 100,0 & & 100,0 & & & 0,0 & & 100,0 & & 100,0 & \\
\hline Com & 3,5 & & -. & 4,1 & $\therefore$ & & 6,7 & .. & 3,6 & .. & 3,7 & .. & 7,5 & $-1,7$ & 4,5 & .. & & 7,4 & .. & 9,9 & 22,8 * & 6,8 & $14,9^{* * *}$ \\
\hline Com Motocicl & 15,7 & & .. & 16,3 & $\therefore$ & & 23,5 & $18,6^{* * *}$ & 19,6 & .. & 18,5 & -. & 10,6 & 5,3 & 9,0 & 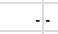 & & 6,2 & -. & 6,7 & $-4,0$ & 14,0 & 10,9 ** \\
\hline Com Carro e M & 0,0 & & -. & 0,0 & $\therefore$ & & 0,2 & .. & 7,1 & .. & 0,0 & .. & 0,0 & .. & 2,2 & .. & & 0,0 & .. & 1,5 & .. & 1,0 & .. \\
\hline Sem Carro e/ou Motocicleta & 81,0 & $-1,0$ & & 79,6 & $-3,0$ & & 69,5 & $-5,4^{* * *}$ & 69,6 & $-4,7^{* * *}$ & 77,8 & $-3,6$ & 81,9 & $-0,7$ & 84,3 & $-2,4$ & & 6,5 & $-4,8^{* * *}$ & 81,9 & $-1,5$ & 78,3 & $-2,5^{* * *}$ \\
\hline
\end{tabular}

Fonte: Microdados das PNADs/IBGE. Elaboração dos autores.

Nota: Os dois traços (- -) significam os tipos de família com menos de 6 observações na amostra. Já (a) é estimativa do coeficiente de uma regressão log-linear contra o tempo. Neste caso, o teste t indica a existência ou não de uma tendência nos dados. Os asteriscos $(* * *, * *, *)$, por sua vez, representam os níveis de significância de $5 \%(* * *), 10 \%(* *)$ e $20 \%(*)$.

Nesse contexto, considerando a suposição apresentada (tendência de movimento pendular), os dados registrados na Tabela 7 ajudam a sustentá-la como plausível. Tratam-se de informações sobre um fenômeno relativamente recente de crescimento da utilização de carros e motocicletas pelos residentes rurais nordestinos para seus deslocamentos entre o rural e o urbano (AQUINO; FREIRE, 2018; AQUINO; NASCIMENTO, 2020). No que 
concerne a essa questão, a Tabela 7 mostra o rápido crescimento - generalizado entre as UFs nordestinas - do número de famílias rurais com carro ou motocicleta ou ambos, especialmente entre as famílias pluriativas e não agrícolas, onde os veículos automotores passam a desempenhar um papel fundamental como meio de transporte e de trabalho.

Outro elemento que contribui para facilitar e aumentar o contato dos residentes rurais com a dinâmica urbana (trabalho, educação, comércio, serviços em geral, etc.), favorecendo o movimento pendular entre o campo e as sedes municipais, conforme anteriormente referido, concerne ao crescente avanço do acesso à telefonia móvel por parte das famílias (e pessoas) residentes nas áreas rurais das UFs nordestinas. Acerca dessa questão, a Tabela 8 indica que o uso de celulares cresceu significativamente em todos os grupos familiares analisados, entre 2011 e 2015.

Durante esse período, a expansão da telefonia móvel avançou rapidamente e o contingente de domicílios rurais, em praticamente todas as UFs nordestinas, sem celular diminuiu substancialmente, principalmente entre as famílias pluriativas e não agrícolas que se aproximavam de um grau de cobertura que variava nos estados, em média, entre $80,0 \%$ e um pouco mais de $90 \%$, em 2015. Já entre as famílias agrícolas e, mais expressivamente entre as famílias de não ocupados, o grau relativo de acesso ao celular era menor, porém, com taxas de crescimento também bastante expressivas e significativas.

Tabela 8 - Distribuição e evolução percentual (\%) do número de domicílios rurais "com" e "sem" telefone celular, segundo cada tipo familiar: UFs e Nordeste, 2011 a 2015

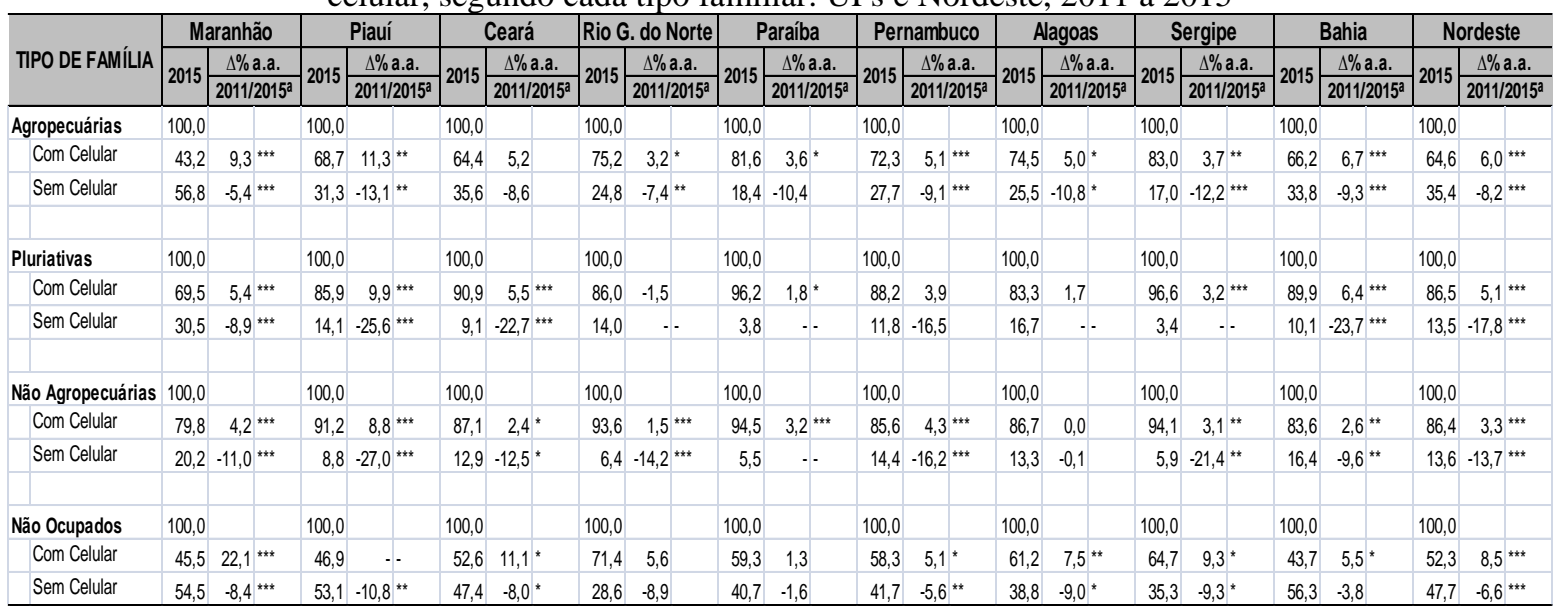

Fonte: Microdados das PNADs/IBGE. Elaboração dos autores.

Nota: Os dois traços (- -) significam os tipos de família com menos de 6 observações na amostra. Já (a) é estimativa do coeficiente de uma regressão log-linear contra o tempo. Neste caso, o teste t indica a existência ou não de uma tendência nos dados. Os asteriscos $(* * *, * *, *)$, por sua vez, representam os níveis de significância de $5 \%(* * *), 10 \%(* *)$ e $20 \%(*)$. 
Evidentemente, vários fatores determinam a capacidade de diversificação das ocupações pelas famílias do campo e seus resultados, desde características individuais das pessoas (sexo, idade, nível educacional), acesso às políticas públicas, até aspectos socioeconômicos do entorno das comunidades rurais (AQUINO et al., 2017; CRUZ et al., 2019; ELLIS, 2000; GOMES DA SILVA; VERAS, 2001; NASCIMENTO, 2009; SCHNEIDER, 2003).

Todavia, a posse de veículos e a telefones móveis potencializa esse processo, contribuindo tanto para apoiar as atividades agrícolas aproximando os produtores e consumidores, como, principalmente, para estimular a pluriatividade e a obtenção de ocupações não agrícolas pelos habitantes do campo, especialmente pelas gerações mais jovens. ${ }^{7}$ Como resultado, gera-se um diferencial de renda entre as famílias estritamente agropecuárias, que tiveram suas receitas reduzidas pelos sucessivos anos de seca, e aquelas que conseguirem acessar outras fontes de trabalho pela maior interação com os ramos industriais, especialmente a construção civil, e com as atividades terciárias desenvolvidas nas cidades da região.

Corroborando o argumento acima, a Tabela 9 evidencia que as maiores rendas médias eram, em 2015, quase que generalizadamente das famílias pluriativas - únicas exceções são os estados do Piauí e Ceará -, seguidas das rendas médias das famílias não agropecuárias. No que respeita às rendas médias das famílias agropecuárias, estas somente ultrapassaram, no ano em questão, as rendas médias das famílias de não ocupados nos estados da PB, PE, AL e BA - nos demais cinco estados as rendas médias das famílias agropecuárias revelaram-se as menores entre os quatro tipos de famílias.

A Tabela 10, por seu turno, complementa as informações anteriores de uma maneira interessante. Note-se, em primeiro lugar, que as rendas médias das famílias agropecuárias só

\footnotetext{
${ }^{7}$ A dinâmica econômica das atividades agropecuárias (especialmente no que diz respeito aos seus rendimentos monetários) apresenta um grau de incerteza mais elevado (comparado às atividades não agropecuárias), o qual é agravado pelas mudanças climáticas. Diante disso, os jovens rurais acostumados a ver as dificuldades enfrentadas por seus pais, sentem-se impelidos a abandonarem o campo em busca de maior segurança em relação à sua vida profissional, o que tende a agravar o problema da sucessão geracional. Nesse sentido, quanto mais o desenvolvimento da infraestrutura regional de transportes e comunicações se direciona para facilitar o contato entre as áreas rurais e urbanas - quanto mais o desenvolvimento aproxima essas duas áreas , menor pode vir a ser o anseio de o jovem querer abandonar o rural, passando a vê-lo com outros olhos, como um espaço de possibilidades de vida, ocupação, trabalho, geração de renda, assim como também (na ausência daquelas possibilidades no rural) de fácil acesso a essas dinâmicas no ambiente urbano. Em outras palavras, quanto mais intenso e facilitado, portanto, o movimento pendular entre o rural e o urbano, ao qual havíamos nos referido anteriormente, menor tende a ser o êxodo, à medida que essa sinergia assegura perspectivas de ocupação e renda mais seguras e estáveis aos jovens rurais, que os tornam simultaneamente mais "pluriativos e plurilocais" (WHITE, 2020).
} 
ganham, em apenas quatro UFs rurais nordestinas, das rendas médias das famílias de não ocupados (Tabela 9). No restante delas, de maneira generalizada em todas as UFs, a elevada dependência das rendas médias das famílias agropecuárias das fontes de rendas do não trabalho (aposentadorias/pensões e outras fontes). Ou seja, o peso das atividades agropecuárias na composição da renda média das famílias agropecuárias não alcança 50,0\% em nenhuma UF rural nordestina - nos casos de Piauí e Ceará não chegam a alcançar 1/4 da renda média familiar, e no Maranhão e na Paraíba alcança apenas um pouco mais de 30,0\%.

Tabela 9 - Renda média dos diferentes tipos de famílias rurais segundo o ramo de atividade: UFs e Nordeste,

\begin{tabular}{|c|c|c|c|c|}
\hline \multirow[b]{2}{*}{ UFs Nordestinas } & \multicolumn{4}{|c|}{2015 (Em R\$ de setembro de/2015) } \\
\hline & $\begin{array}{c}\text { Agropecuárias } \\
\text { (R\$) }\end{array}$ & $\begin{array}{l}\text { Pluriativas } \\
\text { (R\$) }\end{array}$ & $\begin{array}{c}\text { Não } \\
\text { Agropecuárias } \\
(R \$)\end{array}$ & $\begin{array}{c}\text { Não Ocupadas } \\
\text { (R\$) }\end{array}$ \\
\hline Maranhão & 928 & 1.739 & 1.637 & 1.023 \\
\hline Piauí & 964 & 1.735 & 1.914 & 1.120 \\
\hline Ceará & 899 & 1.484 & 1.533 & 949 \\
\hline Rio Gde Norte & 1.129 & 1.787 & 1.692 & 1.259 \\
\hline Paraíba & 1.091 & 1.865 & 1.492 & 956 \\
\hline Pernambuco & 1.070 & 1.785 & 1.358 & 1.035 \\
\hline Alagoas & 1.068 & 1.779 & 1.523 & 903 \\
\hline Sergipe & 929 & 1.687 & 1.463 & 1.016 \\
\hline Bahia & 1.116 & 1.767 & 1.451 & 1.077 \\
\hline Nordeste & 1.021 & 1.723 & 1.539 & 1.022 \\
\hline
\end{tabular}

Fonte: Microdados das PNADs/IBGE. Elaboração dos autores.

Por outro lado, bem diferente das famílias agropecuárias, o peso das fontes de renda do não trabalho na composição da renda média das famílias pluriativas e não agropecuárias é consideravelmente inferior à contribuição das rendas do trabalho, com maior destaque para o caso das famílias não agropecuárias. Já no caso das famílias pluriativas, se considerarmos apenas as rendas do trabalho agropecuário, análogo ao caso das famílias agropecuárias, as rendas do não trabalho têm um peso maior em praticamente quase todas as UFs - diferentemente das rendas não agropecuárias que foram as que mais contribuíram para a composição da renda média das famílias pluriativas.

Torna-se evidente, conforme argumentam Aquino e Nascimento (2020), que a reprodução social das famílias rurais do Nordeste durante a Grande Seca foi influenciada diretamente pelas rendas advindas das aposentadorias rurais e pensões. Assim sendo, vale registrar que apenas no primeiro ano do mencionado fenômeno climático, em 2012, o sistema pagou $\mathrm{R}$ \$ 29,3 bilhões aos seus beneficiários rurais distribuídos nos estados da região (CARVALHO, 2014). Já outra política social relevante foi o Programa Bolsa Família 
(PBF), que, em 2015, emitiu 2,4 milhões de benefícios rurais e injetou mais de $\mathrm{R} \$ 5,6$ bilhões na economia regional (SILVEIRA et al., 2016). Juntas, elas contribuíram diretamente para aumentar a resiliência das famílias pobres e aliviar o peso dos "fatores de expulsão" decorrentes da estiagem prolongada.

Tabela 10 - Composição (\%) da renda média familiar rural, segundo o tipo de família e as diferentes fontes de renda do trabalho (agrícola e não agrícola) e do não trabalho (aposentadorias/pensões e outras fontes): UFs e Nordeste, 2015

\begin{tabular}{|c|c|c|c|c|c|c|c|c|c|c|c|c|c|c|c|c|}
\hline \multirow{5}{*}{ UFs Nordestinas } & \multicolumn{16}{|c|}{ TIPOS DE FAMÍLIAS / TIPOS DE FONTES DE RENDA } \\
\hline & \multicolumn{4}{|c|}{ Agropecuária } & \multicolumn{4}{|c|}{ Pluriativa } & \multicolumn{4}{|c|}{ Não Agropecuária } & \multicolumn{4}{|c|}{ Não Ocupados } \\
\hline & \multicolumn{2}{|c|}{$\begin{array}{l}\text { RENDAS DO } \\
\text { TRABALHO }\end{array}$} & \multicolumn{2}{|c|}{\begin{tabular}{|c|} 
RENDAS DO \\
NÃO TRABALHO
\end{tabular}} & \multicolumn{2}{|c|}{$\begin{array}{l}\text { RENDAS DO } \\
\text { TRABALHO }\end{array}$} & \multicolumn{2}{|c|}{\begin{tabular}{|c|} 
RENDAS DO \\
NÃO TRABALHO
\end{tabular}} & \multicolumn{2}{|c|}{$\begin{array}{l}\text { RENDAS DO } \\
\text { TRABALHO }\end{array}$} & \multicolumn{2}{|c|}{\begin{tabular}{|c|} 
RENDAS DO \\
NÃO TRABALHO
\end{tabular}} & \multicolumn{2}{|c|}{$\begin{array}{l}\text { RENDAS DO } \\
\text { TRABALHO }\end{array}$} & \multicolumn{2}{|c|}{$\begin{array}{c}\text { RENDAS DO } \\
\text { NÃO TRABALHO }\end{array}$} \\
\hline & A & B & C & D & A & B & C & D & A & B & $C$ & D & A & B & $C$ & D \\
\hline & $\%$ & $\%$ & $\%$ & $\%$ & $\%$ & $\%$ & $\%$ & $\%$ & $\%$ & $\%$ & $\%$ & $\%$ & $\%$ & $\%$ & $\%$ & $\%$ \\
\hline Maranhão & 34,8 & -- & 46,8 & 18,5 & 18,5 & 48,7 & 21,4 & 11,4 & -- & 79,6 & 13,5 & 7,0 & -- & -- & 77,2 & 22,8 \\
\hline Piauí & 22,7 & -- & 60,4 & 16,9 & 13,6 & 49,2 & 24,2 & 13,0 & -- & 79,9 & 15,0 & 5,1 & -- & -- & 88,0 & 12,0 \\
\hline Ceará & 21,6 & -- & 53,8 & 24,6 & 18,4 & 40,5 & 27,3 & 13,9 & - & 70,0 & 21,6 & 8,4 & -- & -- & 80,5 & 19,5 \\
\hline Rio Gde N & 43,4 & -- & 37,4 & 19,2 & 26,0 & 47,2 & 17,6 & 9,2 & -- & 78,5 & 13,6 & 8,0 & -- & -- & 53,4 & 46, \\
\hline Paraiba & 33,5 & -- & 50,8 & 15,6 & 16,3 & 51,3 & 21,9 & 10,4 & -- & 72,7 & 17,1 & 10,2 & -- & -- & 85,4 & 14,6 \\
\hline Pernambuco & 43,2 & -- & 40,4 & 16,4 & 22,6 & 47,1 & 19,3 & 11,0 & -- & 75,0 & 16,6 & 8,4 & -- & -- & 80,8 & 19,2 \\
\hline Alagoas & 42,0 & -- & 37,0 & 21,1 & 18,2 & 50,7 & 23,3 & 7,7 & -- & 68,8 & 18,2 & 12,9 & -- & -- & 75,5 & 24,5 \\
\hline Sergipe & 41,8 & -- & 38,7 & 19,5 & 29,8 & 41,0 & 20,4 & 8,8 & -- & 75,3 & 15,7 & 9,0 & -- & -- & 76,5 & 23,5 \\
\hline Bahia & 39,9 & -- & 44,8 & 15,2 & 30,0 & 41,6 & 17,5 & 10,9 &.- & 75,9 & 14,8 & 9,3 & $\ldots$ & $\ldots$ & 84,8 & 15,2 \\
\hline Nordeste & 35,8 & -- & 46,3 & 17,9 & 22,3 & 45,4 & 21,0 & 11,3 & -- & 75,4 & 16,2 & 8,4 & -- & -- & 79,8 & 20,2 \\
\hline
\end{tabular}

Legenda: A: Renda Agropecuária; B: Renda Não-agropecuária; C: Renda de Aposentadoria/Pensão; D: Outras Fontes de Renda.

Fonte: Microdados das PNADs/IBGE. Elaboração dos autores.

Além disso, tais rendas "não dependentes da chuva" e pagas mensalmente também exerceram um efeito multiplicador significativo nas economias urbanas dos pequenos e médios municípios nordestinos, favorecendo (in)diretamente as famílias rurais. De fato, os gastos regulares de consumo do público atendido pelas aposentadorias e pelo PBF contribuem para manter o dinamismo das micro e pequenas indústrias locais de bens populares, do comércio e dos serviços, setores que, como foi visto ao longo do texto, serviram de fonte de ocupação básica para os contingentes crescentes de famílias pluriativas e não agrícolas do campo.

Todos os aspectos mencionados irão se somar às políticas públicas implementadas pelo Estado brasileiro de melhoria da oferta hídrica, de universalização da eletricidade, de pavimentação de estradas e de expansão da construção civil puxada pelo Programa Minha Casa, Minha Vida. Na mesma linha, é preciso acrescentar também os programas de desenvolvimento rural (PRONAF, PAA, PNAE etc.) voltados ao apoio do numeroso 
segmento de agricultores familiares que habita os rurais do Nordeste (AQUINO et al., 2017; MELLO, 2018; MIRANDA; TORRENS; MATTEI, 2017).

Esse conjunto articulado de elementos, como concluem Aquino e Nascimento (2020), contribuiu para tornar o espaço regional mais habitável, ampliando a capacidade de resistência da população rural aos fenômenos climáticos extremos, como foi verificado durante a Grande Seca. Se tal cenário de maior estabilidade social foi afetado ou não pela crise econômica que atingiu o Brasil e os estados da região a partir de 2015/2016, é uma questão importante que deverá ser investigada futuramente.

\section{CONSIDERAÇÕES FINAIS}

O objetivo central do presente estudo consistiu em mostrar que os impactos da última Grande Seca no Nordeste foram bastante distintos dos impactos que esses eventos climáticos provocavam no passado na região, pelo menos no que diz respeito à permanência das pessoas e famílias em suas áreas rurais, assim como também em relação ao perfil da ocupação e fontes de renda das mesmas.

Se durante o século XX obras literárias e diagnósticos técnicos especializados denunciavam que as secas eram motivo de migração das populações atingidas e de mortes pela fome, reduzindo os contingentes rurais, o presente estudo mostrou que na última seca correspondente ao período analisado (2012 a 2015) -, comparativamente aos casos anteriores, nenhuma UF rural nordestina registrou redução de seus contingentes de populacionais. Pelo contrário, todas as taxas foram positivas, observando-se certa convergência entre as respectivas UFs.

Desse modo, as teses sobre a inevitabilidade do esvaziamento demográfico do campo nordestino parecem não ter sustentação ou necessitam ser relativizadas. Isso porque os dados analisados indicam que as áreas rurais da região estão cada vez mais sendo consideradas locais de residência para grupos crescentes de famílias (e pessoas), que pouco ou nenhum vínculo produtivo direto têm com as atividades estritamente agropecuárias (caso das famílias pluriativas, não agrícolas e de não ocupados). Ou seja, o que vem ocorrendo é um processo marcante de "êxodo agrícola" e não a saída definitiva da população do campo.

Obviamente, as taxas de crescimento (quantidades de famílias e pessoas) são relativamente distintas entre os estados, assim como também se pode observar que os 
fatores levantados como possíveis explicações para essas taxas de crescimento também não são totalmente idênticos, o que evidencia a heterogeneidade socioterritorial existente no âmbito regional. Porém, vale ressaltar que, a despeito disso, observou-se, por outro lado, uma razoável homogeneidade entre as UFs relativa à contribuição que as ocupações e rendas obtidas em atividades não agropecuárias deram para manter as pessoas nas áreas rurais, ajudando a enfrentar o longo período de seca.

Quanto às populações de não ocupados, há também uma variedade de situações entre as UFs nordestinas no tocante às suas taxas de crescimento. Assim como também se observou que existe uma forte participação relativa das rendas do não trabalho (aposentadorias/pensões e outras fontes) na composição das rendas familiares, particular e expressivamente entre as famílias estritamente agropecuárias de praticamente todas as UFs, com raras exceções.

Portanto, registra-se uma marcante heterogeneidade entre as famílias rurais nordestinas, no que concerne as suas fontes de ocupação e renda. Tal processo, que apresenta dinâmicas diferenciadas entre os estados da região, tem sido facilitado no período recente pela maior sinergia entre as áreas rurais e urbanas proporcionada pela democratização dos meios de transporte e de comunicação, bem como pela rede de proteção criada pelas políticas públicas de transferência de renda e de desenvolvimento rural.

É razoável supor, contudo, que em outros trabalhos, a partir de uma análise mais desagregada ao nível municipal, seja possível revelar uma diversidade ainda maior de situações socioeconômicas. De qualquer modo, os dados apresentados são suficientes para demonstrar que o campo nordestino não é um espaço homogêneo e nem lugar exclusivo da agropecuária. Sendo assim, os temas da diversificação das estratégias de reprodução social das famílias rurais e das múltiplas funções do rural, como lugar de produção e moradia, não podem ficar de fora das grades de pesquisa dos estudiosos da economia regional e devem figurar como prioridades na agenda dos formuladores de políticas públicas para o desenvolvimento sustentável da região.

\section{REFERÊNCIAS}

AQUINO, J. R. et al. Articulação de políticas para a superação da pobreza rural: um estudo das interfaces entre o Programa Bolsa Família e as políticas de inclusão produtiva no Nordeste e no Sul do Brasil. Revista Econômica do Nordeste. Fortaleza, v. 48, n. 4, p. 155-173, out./dez., 2017. 
AQUINO, J. R.; FREIRE, J. A. A crescente presença das motocicletas no rural nordestino. Jornal O Mossoroense, Mossoró/RN, 26 de abril de 2018, p. 11. Disponível em: $<$ http://www.omossoroense.com.br/artigo-a-crescente-presenca-das-motocicletas-no-ruralnordestino/>. Acesso em: 06 mar. 2019.

AQUINO, J. R.; NASCIMENTO, C. A. O "novo" rural do Rio Grande do Norte revisitado. Cadernos de Ciências Sociais Aplicadas. Vitória da Conquista/BA, n. 20, p.135-157, 2015.

AQUINO, J. R.; NASCIMENTO, C. A. A Grande Seca e as fontes de ocupação e renda das famílias rurais no Nordeste do Brasil. Revista Econômica do Nordeste. Fortaleza, 2020 (No prelo).

ARAÚJO, T. B. Nordeste, Nordestes: que Nordeste? In: ARAÚJO, T. B. Ensaios sobre o desenvolvimento brasileiro: heranças e urgências. Rio de Janeiro: Revan/Fase, 2000. p. 165-196.

ARAÚJO, T. B. (Coord.). Concepções da ruralidade contemporânea: as singularidades brasileiras. Brasília, IICA, 2013. (Série Desenvolvimento Rural Sustentável; v. 21).

ARAÚJO, T. B. Nordeste: desenvolvimento recente e perspectivas. In: GUIMARÃES, P. F. et al. (Orgs.). Um olhar territorial para o desenvolvimento: Nordeste. Rio de Janeiro: BNDES, 2014. p. 540-560.

BURITI, C. O.; BARBOSA, H. A. Um século de secas: porque as políticas hídricas não transformaram o semiárido brasileiro? Lisboa/Portugal: Editora Chiado, 2018.

CARVALHO, C. P. O. O novo padrão de crescimento no Nordeste semiárido. Revista Econômica do Nordeste. Fortaleza/CE, v.45, n. 3. p. 160-184, jul./set. 2014.

CRUZ, M. P. M. et al. Diferenciais de rendimentos entre atividades agrícolas e não agrícolas no meio rural nordestino. Revista de Desenvolvimento Econômico. Salvador/BA, v. 2, n. 43, p. 201-231, ago./2019.

ELLIS, F. Rural livelihoods and diversity in developing countries. Oxford: Oxford University Press, 2000.

FURTADO, C. Seca e poder. São Paulo: Ed. Fundação Perseu Abramo, 1998.

GOMES DA SILVA, A. Novas configurações no espaço rural do Rio Grande do Norte. In: CAMPANHOLA, C.; GRAZIANO DA SILVA, J. (Eds.). O novo rural brasileiro: uma análise estadual - Nordeste. Jaguariúna, SP: EMBRAPA, 2000. p. 13-44.

GOMES DA SILVA, A.; VERAS, E. C. A heterogeneidade da dinâmica das ocupações no rural do Nordeste. In: CONGRESSO BRASILEIRO DE ECONOMIA E SOCIOLOGIA RURAL, 39., 2001, Recife/PE. Anais... Recife/PE: SOBER, 2001. (CD-ROM). 
GRAZIANO DA SILVA, J. O novo rural brasileiro. 2. ed. rev. Campinas: Unicamp/IE, 1999.

GTDN. Uma política de desenvolvimento econômico para o Nordeste. 2. ed. Recife: SUDENE, 1967.

GUJARATI, D. N. Econometria básica. 4. ed. Rio de Janeiro: Ed. Campus, 2006.

HOFFMANN, R. Quatro tipos de testes de hipóteses com os dados das PNADs. In: CAMPANHOLA, C.; GRAZIANO DA SILVA, J. (Orgs.). O novo rural brasileiro: uma análise nacional e regional. Jaguariúna: Embrapa, 2000. p. 137-155.

IBGE. Pesquisa Nacional por Amostra de Domicílios - PNAD: 2001 a 2014. Rio de Janeiro: IBGE, 2015. Disponível em: 〈http://www.sidra.ibge.gov.br/pnad/pnadpb.asp>. Acesso em: 20 nov. 2015.

IBGE. MUNIC 2017: perfil dos municípios brasileiros. Rio de Janeiro: IBGE, 2018.

MAIA GOMES, G. Velhas secas em novos sertões: continuidade e mudanças na economia do semi-árido e dos cerrados nordestinos. Brasília: IPEA, 2001.

MAIA GOMES, G. Sertões não-euclidianos. Recife/PE, 2014. 18p. (Texto mimeo).

MARTINS, E. S. P. R.; MAGALHÃES, A. R. A seca de 2012-2015 e seus impactos. Parcerias Estratégicas. Brasília, v. 20, n. 41, p.107-128, jul./dez., 2015.

MATTEI, L. A relevância da família como unidade de análise nos estudos sobre pluriatividade. Revista de Economia e Sociologia Rural. Rio de Janeiro, v. 45, n. 4, p. 1.055-1.073, out./dez. 2007.

MELO NETO, J. C. Morte e vida Severina. Rio de Janeiro: Objetiva, 2007.

MELLO, J. Estratégias de superação da pobreza no Brasil e impactos no meio rural. Rio de Janeiro: IPEA, 2018.

MIRANDA, C.; TORRENS, J.; MATTEI, L. (Orgs.). O legado das políticas públicas de desenvolvimento rural para a inclusão socioprodutiva no Brasil. Brasília: IICA, 2017. (Série Desenvolvimento Rural Sustentável; v. 24).

NASCIMENTO, C. A. A pluriatividade das famílias rurais no Nordeste e no Sul do Brasil: pobreza rural e políticas públicas. Economia e Sociedade. Campinas/SP, v. 18, n. 2 (36), p. 317-348, ago./2009.

NEDER, H. D. A utilização estatística das informações das PNADs: testes de hipóteses e reamostragem. In: CAMPANHOLA, C.; GRAZIANO DA SILVA, J. (Orgs.). O novo rural brasileiro: uma análise nacional e regional. Jaguariúna: Embrapa, 2000. p. 101-136. 
NYS, E; ENGLE, N. L.; MAGALHÃES, A. R. (Orgs.). Secas no Brasil: política e gestão proativas. Brasília: CGEE/Banco Mundial, 2016.

QUEIROZ, R. O quinze. 93. ed. Rio de Janeiro. José Olympio, 2012.

RAMOS, G. Vidas secas. 108. ed. Rio de Janeiro: Record, 2008.

SCHNEIDER, S. A pluriatividade na agricultura familiar. Porto Alegre: Editora da UFRGS, 2003.

SILVEIRA, F. G. et al. Políticas públicas para o desenvolvimento rural e de combate à pobreza no campo. Brasília: IPC-IG/PNUD, 2016.

SOARES, S. et al. Perfil da pobreza: Norte e Nordeste rurais. Brasília: IPC-IG/PNUD, 2016.

WHITE, B. Rural household pluriactivity and plurilocality: a source of resilience to climate breakdown. In: IOP Conference Series: Earth and Environmental Science (451 012001), 2020. 